Pacific Journal of Mathematics

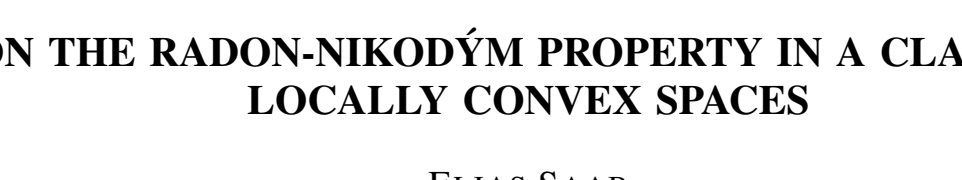




\title{
ON THE RADON-NIKODYM PROPERTY IN A CLASS OF LOCALLY CONVEX SPACES
}

\author{
Elias SAAB
}

In an earlier paper we studied the Radon-Nikodym property (RNP) for Fréchet spaces. D. Gilliam continued the study by examining the RNP for locally convex spaces with the strict Mackey convergence property. The aim of this paper is to take one more step by studying the RNP for the class of locally convex spaces in which every bounded subset is metrizable. Although this class strictly includes the class of spaces with the strict Mackey convergence property, our goal is not a generalization for the sake of generalization. Indeed, we shall prove a theorem that reduces the study of the RNP for this class of spaces directly to the study of the RNP for Banach spaces. This will provide a quick and simultaneous extension of many of the basic Radon-Nikodym theorems in Banach spaces to this class of locally convex spaces. We hope that our technique will eliminate some of the mystery that seems to surround the RNP for locally convex spaces.

1. Definitions and preliminaries. Throughout this paper $(E, \tau)$ will always be a quasi-complete locally convex Hausdorff space in which every bounded subset is metrizable and $\tau$ will denote its topology.

Let $(T, \Sigma, P)$ be a probability space and $m: \Sigma \rightarrow E$ be a vector measure. For every continuous semi-norm $q$ on $E$, the $q$-variation of $m$ over $X$ in $\Sigma$ is defined to be

$$
\begin{aligned}
|m|_{q}(X)=\sup \left\{\sum_{i=1}^{n} q\left(m\left(X_{i}\right)\right) ;\left\{X_{i}\right\}_{i=1}^{n} \text { disjoint, } X_{i} \subset X \text { and } X_{i} \in \Sigma\right. & \text { for } 1 \leqq i \leqq n\} .
\end{aligned}
$$

The function $|m|_{q}$ is an extended real-valued measure. The vector measure $m$ is said to be of bounded variation if $|m|_{q}(T)<+\infty$ for every continuous semi-norm $q$ on $E$. Also $m$ is said to be $P$-continuous (denoted $m \ll P$ ) if $m(X)=0$, whenever $P(X)=0$ and $X \in \Sigma$. It is clear that $m \ll P$ if and only if for every continuous semi-norm $q$ on $E$ we have $|m|_{q} \ll P$. The set

$$
\operatorname{Am}(\Sigma)=\left\{\frac{m(X)}{P(X)} ; X \in \Sigma, P(X)>0\right\}
$$

is called the $P$-average range of $m$. 
Definition 1.1. A function $f: T \rightarrow E$ is said to be $P$-integrable if and only if there exists a sequence $f_{n}$ of simple functions such that:

(i) $\lim _{n} f_{n}(t)=f(t) P$-almost everywhere (P.a.e.)

(ii) $\lim _{n} \int_{T} q\left(f_{n}(t)-f(t)\right) d P=0$ for every continuous semi-norm $q$ on $E$.

This definition allows us to define $\int_{X} f d P=\lim _{n} \int_{X} f_{n} d P$ for each $X$ in $\Sigma$, using the fact that $E$ is quasi-complete.

It can be verified that this definition is independent of the choice of the sequence $\left(f_{n}\right)$, and if $F$ is another quasi-complete locally convex Hausdorff space and $U: E \rightarrow F$ is a continuous linear operator, then $U \circ F$ is also $P$-integrable and $U\left(\int_{X} f d P\right)=\int_{X} U \circ f d P$ for all $X$ in $\Sigma$.

We adopted this definition because all the $P$-integrable functions we will be dealing with take their values in a bounded metrizable set. This definition is equivalent to the one used in [15] when the space $E$ is a Fréchet space.

Definition 1.2. Let $C$ be a closed bounded convex subset of $E$. The set $C$ is said to have the $R N P$ if for every probability space $(T, \Sigma, P)$ and every vector measure $m: \Sigma \rightarrow E$ whose $P$-average range is contained in $C$ there exists a $P$-integrable function $f: T \rightarrow C$ such that $m(X)=\int_{X} f d P$ for every $X$ in $\Sigma$.

If every bounded closed convex subset of $E$ has the RNP, then $E$ is said to have RNP.

Note that in this definition the boundedness of the set $C$ insures that any vector measure whose $P$-average range is contained in $C$ is of finite variation and is $P$-continuous.

For each subset $B$ of $E$, let $\overline{\operatorname{conv}}(B)$ denote the closed convex hull of $B$ and define $s(B)$ to be the set

$$
s(B)=\left\{\sum_{n=1}^{\infty} \lambda_{n} b_{n} ; \lambda_{n}>0, \sum_{n=1}^{\infty} \lambda_{n}=1,\left(b_{n}\right) \subset B \text { and } \sum_{n=1}^{\infty} \lambda_{n} b_{n} \text { converges }\right\} .
$$

The set $s(B)$ is called the $s$-convex hull of $B$. It is clear that $B \subset s(B) \subseteq \operatorname{conv}(B)$.

Definitions 1.3. A subset $B$ of $E$ is said to be dentable $(s$-dentable) if and only if for every zero-neighborhood $V$ in $E$ there exists $b \in B$ such that $b \notin \overline{\operatorname{conv}}(B \backslash(b+V))(b \notin s(B \backslash(b+V)))$.

$A$ set $B$ is said to be subset dentable (subset $s$-dentable) if every subset of $B$ is dentable ( $s$-dentable). 
If $A$ is a bounded subset of $E$, a slice of $A$ is a subset of $A$ defined by

$$
S(f, r, A)=\left\{x \in A ; f(x) \geqq \sup _{A} f-r\right\}
$$

where $f$ is in $E^{*}, f \neq 0$ and $r>0$.

Definition 1.4. A point $x$ in $A$ is said to be denting if for every zero-neighborhood $V$ in $E x \notin \overline{\operatorname{conv}}(A \backslash(x+V))$.

Definition 1.5. A point $x$ in $A$ is said to be exposed if there exists $f \in E^{*}$ such that $f(x)=\sup _{A} f$ and $f(z)<f(x)$ for all $z \in A, z \neq x$.

Definition 1.6. A point $x$ in $A$ is said to be strongly exposed if there exists $f \in E^{*}$ such that for every zero-neighborhood $V$ there exists $r>0$ such that $x \in S(f, r, A)=S$ and $S-S \subset V$.

Before proving the main theorem, we are going to give some examples of locally convex spaces in which every bounded subset is metrizable.

Obviously every Fréchet space and every locally convex space with the strict Mackey convergence property [10] belong to this class. The space $l^{1}$ with its $w^{*}$-topology belongs to this class but does not have the strict Mackey convergence property.

It can be shown that this class is sequentially closed under strict inductive limits: in particular every LF-space belongs to this class.

The results of this paper were announced in [16].

\section{The space $\left(\hat{E}_{M}, N\right)$ : properties and consequences.} Let $C$ be a closed bounded convex subset of $(E, \tau)$, let $M=$ $\overline{\operatorname{conv}}(C \cup-C)$ and let $E_{M}=\cup_{n=1}^{\infty} n M$. Then we have the following theorem.

THEOREM 2.1. There exists a norm $N$ on $E_{M}$ such that the topology induced by $\left(E_{M}, N\right)$ on $M$ coincides with the topology induced by $(E, \tau)$ on $M$.

Proof. There exists a sequence $V_{n}$ of closed absolutely convex zero-neighborhoods in $(E, \tau)$ such that

(1) $V_{n+1}+V_{n+1} \subset V_{n}$ for every $n \geqq 1$.

(2) $\left\{V_{n} \cap(M-M)\right\}_{n \geqq 1}$ forms a fundamental system of zeroneighborhoods in $(M-M, \tau)$.

Let $\tau_{1}$ be the topology on $E$ that has $\left\{V_{n}\right\}_{n \geqq 1}$ as a fundamental system 
of zero-neighborhoods. The topology $\tau_{1}$ is not in general Hausdorff but the restriction of $\tau_{1}$ on $E_{M}$ is Hausdorff and $\tau_{1}$ induces on $M$ the same topology as $\tau$. To see this, let $x \in M$ and let $V$ be a $\tau$-zeroneighborhood in $E$; it is enough to show that $(x+V) \cap N$ contains $\left(x+V_{n}\right) \cap M$ for some $n$.

To this end, note that there exists $n$ such that

$$
V_{n} \cap(M-M) \subset V \cap(M-M)
$$

Let $y \in\left(x+V_{n}\right) \cap M$, then one has $y-x \in V_{n} \cap(M-M)$, accordingly $y-x \in V \cap(M-M)$; hence $y \in(x+V) \cap M$.

Thus $\left(x+V_{n}\right) \cap M \subset(x+V) \cap M$. This proves that the restriction of $\tau$ to $M$ is coarser than the restriction of $\tau_{1}$ to $M$. On the other hand, it is clear that $\tau$ restricted to $M$ is finer than $\tau_{1}$ restricted to $M$. Thus $\tau$ and $\tau_{1}$ agree on $M$.

We now turn to the construction of the norm $N$. Since $M$ is bounded, for every $n$ there exists $a_{n} \geqq 1$ such that $M \subset a_{n} V_{n}$. Let $p_{n}$ be the gauge functional of $V_{n}$. For every $x \in E_{M}$ define

$$
N(x)=\sum_{n=1}^{\infty} \frac{1}{a_{n} 2^{n}} p_{n}(x) \text {. }
$$

It is clear that $N(x)<+\infty$ for every $x \in E_{M}$. If $N(x)=0$ then $p_{n}(x)=0$ for every $n$, this implies that $x=0$ because $\tau_{1}$ is Hausdorff on $E_{M}$. It follows that $N$ is a norm on $E_{M}$, let $\tau_{2}$ be the topology defined by $N$ on $E_{M}$.

To complete the proof it is enough to show that $\tau_{2}$ restricted to $M$ is the same as $\tau_{1}$ restricted to $M$. Evidently $\tau_{1}$ restricted to $M$ is coarser than $\tau_{2}$ restricted to $M$. Conversely let $x \in M$, let

$$
B_{N}(x, \epsilon)=\{y \in M ; N(x-y) \leqq \epsilon\}
$$

and let

$$
B_{k}(x, \epsilon)=\left\{y \in M ; p_{k}(x-y) \leqq \epsilon\right\}
$$

It is enough to prove that:

$$
B_{k}\left(x, \frac{1}{2^{k}}\right) \subset B_{N}\left(x, \frac{3}{2^{k}}\right)
$$

To this end, let $y \in B_{k}\left(x, 1 / 2^{k}\right)$. Note that $p_{1}(x-y) \leqq p_{2}(x-y) \leqq$ $\cdots \leqq p_{k}(x-y) \leqq 1 / 2^{k}$. From this we obtain 


$$
\begin{aligned}
N(x-y) & =\sum_{n=1}^{k} \frac{1}{a_{n} 2^{n}} p_{n}(x-y)+\sum_{n=k+1}^{\infty} \frac{1}{a_{n} 2^{n}} p_{n}(x-y) \\
& \leqq \frac{1}{2^{k}} \sum_{n=1}^{k} \frac{1}{2^{n}}+\sum_{n=k+1}^{\infty} \frac{2 a_{n}}{a_{n} 2^{n}} \\
& =\frac{1}{2^{k}} \sum_{n=1}^{k} \frac{1}{2^{n}}+\frac{1}{2^{k}} \sum_{n=0}^{\infty} \frac{1}{2^{n}} \\
& \leqq \frac{1}{2^{k}}[1+2]=\frac{3}{2^{k}} .
\end{aligned}
$$

One can also easily check that the uniform structure induced by $N$ on $M$ coincides with the uniform structure induced by $\tau$ on $M$ and consequently $M$ is complete in $\left(E_{M}, N\right)$ because it is complete in $(E, \tau)$. Let $\left(\hat{E}_{M}, N\right)$ be the completion of $\left(E_{M}, N\right)$.

As a corollary of Theorem 2.1 we have:

Corollary 2.2. Let $C$ and $M$ be as in Theorem 2.1. Then:

(i) The set $C$ is dentable (s-dentable) in $(E, \tau)$ if and only if $C$ is dentable (s-dentable $)$ in $\left(\hat{E}_{M}, N\right)$.

(ii) A point $x \in C$ is denting in $(E, \tau)$ if and only if $x$ is denting in $\left(\hat{E}_{M}, N\right)$.

Before establishing the relations between dentability, $s$-dentability and the Radon-Nikodym property we need the following theorem.

TheOREM 2.3. Let $(T, \Sigma, P)$ be a probability space and let $C$ and $M$ be as above. Then:

A function $f: T \rightarrow C$ is $P$-integrable in $\left(\hat{E}_{M}, N\right)$ if and only if $f$ is $P$-integrable in $(E, \tau)$. $X$ in $\Sigma$.

In this case $\int_{X} f d P$ in $\left(\hat{E}_{M}, N\right)$ is the same as $\int_{X} f d P$ in $(E, \tau)$ for every

Proof. Suppose that $f$ is $P$-integrable in $\left(\hat{E}_{M}, N\right)$, then there exists a sequence $f_{n}: T \rightarrow C$ of simple functions such that

(i) $\lim _{n} N\left(f_{n}(t)-f(t)\right)=0$ P.a.e. and

(ii) $\lim _{n} \int_{T} N\left(f_{n}(t)-f(t)\right) d P=0$.

By Theorem $2.1 f_{n}(t) \rightarrow f(t) P$.a.e. in $(E, \tau)$. Thus to complete the proof we must show that $\lim _{n} \int_{T} q\left(f_{n}(t)-f(t)\right) d P=0$ for every continuous seminorm $q$ on $(E, \tau)$. For note that although the injection $\left(E_{M}, N\right) \rightarrow(E, \tau)$ is not necessarily continuous, its restriction to $M$ is 
continuous by Theorem 2.1. Consider the sequence $h_{n}(t)=$ $q\left(f_{n}(t)-f(t)\right)$. This sequence is a real valued sequence of uniformly bounded integrable functions which tends to zero $P$.a.e. By an appeal to the bounded convergence theorem, we have $\lim _{n} \int_{T} q\left(f_{n}(t)-f(t)\right) d P=0$.

Conversely, suppose that $f: T \rightarrow C$ is $P$-integrable in $(E, \tau)$. Consider the sequence $p_{n}$ which defines the topology $\tau_{1}$ on $E_{M}$ (see Theorem 2.1) with the help of ([9], p. 241), choose for every $n \geqq 1$ a sequence $\left(\phi_{k}^{n}\right)_{k \geqq 1}$ of simple functions from $T$ to $C$ such that

$$
\lim _{k} p_{n}\left(\phi_{k}^{n}(t)-f(t)\right)=0 \text { P.a.e. }
$$

By the bounded convergence theorem, we have

$$
\lim _{k} \int_{T} p_{n}\left(\phi_{k}^{n}(t)-f(t)\right) d P=0
$$

for every $n \geqq 1$.

By ([9], p. 254), one can find a sequence $f_{k}: T \rightarrow C$ of simple functions such that $\lim _{k} \int_{T} p_{n}\left(f_{k}(t)-f(t)\right) d P=0$ for every $n \geqq 1$. Now use the diagonal process to choose a sequence $g_{n}: T \rightarrow C$ of simple functions that converges to $f P$.a.e. for the topology $\tau_{1}$. This proves that $g_{n}$ converges to $f P$.a.e. in $\left(E_{M}, N\right)$, and thus proves that $f$ is $P$-measurable in $\left(\hat{E}_{M}, N\right)$. Since $f$ is bounded in $\left(\hat{E}_{M}, N\right)$ this proves that $f$ is $P$ integrable in $\left(\hat{E}_{M}, N\right)$.

Corollary 2.4. Let $C$ and $M$ be as above. Then $C$ has the RNP in $(E, \tau)$ if and only if $C$ has the RNP in $(E, N)$.

Now Corollary 2.2 and Corollary 2.4 together with results of Rieffel [14], Maynard [12], Davis-Phelps [4] and Huff [11] (see [5] and [6]) for Banach spaces prove the following result:

THEOREM 2.5. Let $C$ be a closed bounded convex subset of $E$, then the following assertions are equivalent:

(i) The set $C$ has the RNP.

(ii) The set $C$ is subset dentable.

(iii) The set $C$ is subset $s$-dentable.

We now pass to the discussion of the existence of denting points in a closed bounded convex subset of $E$. 
Phelps [13] showed that if $F$ is a Banach space such that every subset of $F$ is dentable then every closed bounded convex subset of $F$ is the closed convex hull of its strongly exposed points. Phelps's argument is global in nature and does not seem to give local information about subset dentable closed bounded convex sets in arbitrary Banach spaces. J. Johnson and J. Bourgain have independently shown that the following theorem is a consequence of a recent paper of Bourgain [1].

THEOREM 2.6. Let $F$ be a Banach space and $C$ be a closed convex bounded subset of $E$ having the RNP then $C$ is the closed convex hull of its strongly exposed points.

Now using this theorem together with Corollary 2.2 and Corollary 2.4 we can prove the following theorem.

THEOREM 2.7. Let $C$ be a closed bounded convex subset of $(E, \tau)$. Then the following assertions are equivalent:

(i) The set $C$ has the RNP.

(ii) Every closed convex subset of $C$ is the closed convex hull of its denting points.

Proof. (i) $\Rightarrow$ (ii) Let $\quad M=\overline{\operatorname{conv}}(C \cup-C)$ and consider $M \subset\left(\hat{E}_{N}, N\right)$. Let $C_{1}$ be a closed convex subset of $C$. Then $C_{1}$ has the RNP in $(E, \tau)$ and therefore $C_{1}$ has the RNP in $\left(\hat{E}_{M}, N\right)$. By Theorem 2.6, $C_{1}$ is the closed convex hull of its strongly exposed points in $\left(\hat{E}_{M}, N\right)$ and in particular of its denting points in $\left(\hat{E}_{M}, N\right)$. An appeal to Theorem 2.1 and Corollary 2.2 finishes the proof.

The other implication is immediate from the definitions and Theorem 2.5.

It is natural to ask whether one can replace denting points by strongly exposed points in Theorem 2.7.

The answer is no. Consider

$$
C=[-1,1]^{N}
$$

The set $C$ is a convex compact set in the Fréchet space $F$, but from the fact that $F^{*}$ consists of the finitely nonzero sequences, it is easily seen that $C$ does not even have any exposed points.

\section{The Radon-Nikodym theorem, Dunford-Pettis-} Phillips theorem, Liaponouv-Uhl's theorem and Edgar's theorem. Now we will use the well known results in Banach spaces and what we did before to deduce the following Radon-Nikodym theorem. Before doing this let us recall one definition. 
Definition 3.1. Let $(T, \Sigma, P)$ be a probability space and $m: \Sigma \rightarrow E$ be a vector measure. The measure $m$ is said to have a locally relatively compact (relatively weakly compact,...) $P$-average range if and only if for every $\epsilon>0$ there exists $T_{\epsilon} \subset T$ such that $P\left(T \backslash T_{\epsilon}\right) \leqq \epsilon$ and the set

$$
\left\{\frac{m(X)}{P(X)} ; X \in \Sigma, X \subset T_{\epsilon}, P(X)>0\right\}
$$

is relatively compact (relatively weakly compact,...).

THEOREM 3.2. Let $(T, \Sigma, P)$ be a probability space and $m: \Sigma \rightarrow(E, \tau)$ be a vector measure with bounded $P$-average range then the following assertions are equivalent:

(i) The measure $m$ has a locally relatively compact $P$-average range.

(ii) The measure $m$ has a locally relatively weakly compact $P$ average range.

(iii) The measure $m$ has a locally dentable $P$-average range.

(iv) The measure $m$ has a locally $s$-dentable $P$-average range.

(v) There exists $f: T \rightarrow E P$-integrable such that $m(X)=\int_{X} f d P$ for every $X \in \Sigma$.

Proof. We reduce the proof to the case of Banach spaces by considering

$$
M=\overline{\operatorname{conv}}(\operatorname{Am}(\Sigma) U-\operatorname{Am}(\Sigma))
$$

and everything can be studied inside $M$ considered as a subset of the Banach space $\left(\hat{E}_{M}, N\right)$. With this in mind apply Theorem 2.1, Corollary 2.2, Theorem 2.3 and the results in Banach spaces [6] to complete the proof.

Before proving a theorem of Dunford-Pettis-Phillips type we need the following proposition which can be proved using Smulian's theorem, ([7] p. 433) and Theorem 2.1.

Proposition 3.3. Let $C$ and $M$ be as in the Theorem 2.1. Then $C$ is weakly compact in $(E, \tau)$ if and only if $C$ is weakly compact in $\left(\hat{E}_{M}, N\right)$.

The following result shows that the Dunford-Pettis-Phillips theorem is valid in the class of spaces $E$ under consideration in this paper.

Proposition 3.4. For every weakly compact operator $W: L^{1}[0,1] \rightarrow(E, \tau)$ there exists $g:[0,1] \rightarrow(E, \tau) \lambda$-integrable $(\lambda$. the 
Lebesgue measure of $[0,1])$ such that $W(f)=\int_{0}^{1} f g d \lambda$ for every $f$ in $L^{1}[0,1]$, and in particular $W$ sends weakly relatively compact sets into relatively $\tau$-compact sets.

Proof. Let $M$ be the $\tau$-closure of the image of the unit ball of $L^{1}[0,1]$ by $W$, now $M$ is weakly compact in $(E, \tau)$ and therefore it is weakly compact in $\left(\hat{E}_{M}, N\right)$ by Proposition 3.4. By the Dunford-Pettis-Phillips theorem there exists $g:[0,1] \rightarrow M \lambda$-Bochner integrable in $\left(\hat{E}_{M}, N\right)$ such that $W(f)=\int_{0}^{1} f g d \lambda$ for every $f$ in $L^{1}[0,1]$. It is easy to see that the function $t \rightarrow f(t) g(t)$ is $\lambda$-integrable from $[0,1] \rightarrow(E, \tau)$ and $W(f)=\int_{0}^{1} f g d \lambda$ in $(E, \tau)$.

The following theorem was proven by Uhl [18] in the case of Banach space. It is a Liapounov type theorem.

THEOREM 3.5. Let $E$ have the RNP and let $m: \Sigma \rightarrow E$ be a non atomic vector measure with bounded $P$-average range, then the closure of the range of $m$ is convex and compact.

Proof. Let $M=\overline{\operatorname{conv}}(\operatorname{Am}(\Sigma) U-\operatorname{Am}(\Sigma))$. As usual we consider $M$ as a subset of $\left(\hat{E}_{M}, N\right)$. Note that $m: \Sigma \rightarrow M$ is a vector measure when $M$ is considered as a subset of $\left(\hat{E}_{M}, N\right)$. Since $M$ has the RNP in $(E, \tau)$, then it has the RNP in $\left(\hat{E}_{M}, N\right)$ by the Corollary 2.4. Therefore there exists $f: T \rightarrow M P$-integrable such that $m(X)=\int_{X} f d P$ for every $X$ in $\Sigma$. As in Uhl [18] we obtain that the closure of $m(\Sigma)$ is convex and compact in $\left(\hat{E}_{M}, N\right)$. But this closure is a subset of $M$. Thus it is also compact in $(E, \tau)$ by Theorem 2.1 .

In [8] Edgar established a representation theorem of Choquet type [3] for a bounded convex separable subset $C$ of a Banach space when $C$ has the RNP. We are going to show that Edgar's theorem is also valid in the locally convex spaces under consideration.

We refer the reader to [8], for the notations and terminology used in the sequel.

THEOREM 3.6. Let $C$ be a bounded closed convex separable subset of $(E, \tau)$ having the $R N P$. Then for every $a \in C$ there exists a probability measure $\mu$ on the universally measurable subsets of $C$ such that $\mu(\operatorname{Ext}(C))=1$ and $\int_{C} x d \mu=a$ in $(E, \tau)(\operatorname{Ext}(C)$ is the set of extreme points of $C$ ). 
Proof. Let $M=\overline{\operatorname{conv}}(C \cup-C)$ and consider $C$ in $\left(\hat{E}_{M}, N\right)$. Since $C$ has the RNP in $(E, \tau)$, the set $C$ has the RNP in $\left(\hat{E}_{M}, N\right)$. Now by Edgar's theorem there exists a probability measure $\mu$ defined on the universally measurable subsets of $C$ such that $\mu(\operatorname{Ext}(C))=1$ and $\int_{C} x d \mu=a$ in $\left(\hat{E}_{M}, N\right)$. Therefore by Theorem $2.3 \int_{C} x d \mu=a$ in $(E, \tau)$.

The uniqueness theorem (see [17] and [2]) can also be deduced using the space $\left(\hat{E}_{M}, N\right)$ to obtain:

THEOREM 3.7. Under the same hypothesis as the above theorem: the following assertions are equivalent:

(i) The set $C$ is a simplex.

(ii) For every $a \in C$ there exists a unique probability measure $\mu$ on the universally measurable subsets of $C$, such that $\int_{C} x d \mu=a$ and $\mu(\operatorname{Ext}(C))=1$.

We finish by asking the following:

Problem. Let $F$ be a locally convex Hausdorff space and let $C$ be a bounded closed convex metrizable subset of $F$, is $M=\overline{\operatorname{conv}}(C \cup-C)$ metrizable?

If the answer is yes, then Theorem 2.1 and consequently Theorem 2.5, Theorem 2.7, Theorem 3.6 and Theorem 3.7 will be true if we suppose only that $C$ is a metrizable subset of an arbitrary quasi-complete locally convex Hausdorff space.

The author is happy to acknowledge helpful discussions with Professor J. J. Uhl.

\section{REFERENCES}

1. J. Bourgain, On dentability and the Bishop-Phelps property, (preprint).

2. R. D. Bourgain and G. A. Edgar, Non-compact simplexes in Banach spaces with the Radon-Nikodym property, J. Functional Analysis, 23 (1976), 162-178.

3. G. Choquet and P. A. Meyer, Existence et unicité des représentation integrales dans les convexes compacts quelconques, Ann. Inst. Fourier, 13 (1963), 139-154.

4. W. J. Davis and R. R. Phelps, The Radon-Nikodym property and dentable sets in Banach space, Proc. Amer. Math. Soc., 45 (1974).

5. J. Diestel and J. J. Uhl Jr., The Radon-Nikodym theorem for Banach space valued measures, Rocky Mountain J. Math., 6, No. 1 (1976).

6. — Vector measures, Math. Surveys no. 15, Amer. Math. Soc. Providence, 1977.

7. N. Dunford and J. T. Schwartz, Linear Operators, Part I., Interscience, New York, (1958).

8. G. A. Edgar, A non compact Choquet theorem, Proc. Amer. Math. Soc., 49 (1975), 354-358.

9. H. G. Garnir, M. De Wilde and J. Schmets, Analyse Fonctionelle, $t$ II, Birkhauser Verlag (1968). 
10. D. Gilliam, Geometry and the Radon-Nikodym Theorem in strict Mackey convergence spaces, Pacific J. Math., (to appear).

11. R. E. Huff, Dentability and the Radon-Nikodym property, Duke Math. J., 41 (1974), 111-114.

12. H. B. Maynard, A geometric characterization of Banach spaces with the Radon-Nikodym property, J. Functional Analysis, 185 (1973), 493-500.

13. R. R. Phelps, Dentability and extreme points in Banach spaces, J. Functional Analysis, 17 (1974), 78-90.

14. M. A. Rieffel, Dentable subsets of Banach spaces, with application to a Radon-Nikodym theorem. "Functional Analysis", Washington, Thompson Book company, (1967), 71-77.

15. E. Saab, Dentabilité. points extrémaux et propriété de Radon-Nikodym, Bull. Sci. Math, 2ème Série, 99 (1975), 129-134.

16. - Sur la proprieté de Radon-Nikodym dans les espaces localement convexe de type (BM),

C. R. Acad. Sc. Paris, Serie A (1976), 899-902.

17. J. Saint-Raymond, Représentation intégrale dans certains convexes, Séminaire Choquet (1975).

18. J. J. Uhl Jr., The range of a vector-valued measure, Proc. Amer. Math. Soc., 23 (1969), 158-163.

Received August 16, 1976 and in revised form April 20,1977. This work was partially supported by the NCSR-LEBANON, and will constitute a portion of the author's Ph.D. thesis at the University of Illinois.

UNIVERSITY OF ILLINOIS

URBANA, IL 61801 



\section{PACIFIC JOURNAL OF MATHEMATICS EDITORS}

ICHARD ARENS (Managing Editor)

niversity of California

os Angeles, CA 90024

\section{. A. BEAUmont}

niversity of Washington

sattle, WA 98105

. C. MOORE

niversity of California

erkeley, CA 94720
J. DUGUNDJI

Department of Mathematics

University of Southern California

Los Angeles, CA 90007

R. FINN AND J. MiLgRAM

Stanford University

Stanford, CA 94305

\section{ASSOCIATE EDITORS}
F. BECKENBACH
B. H. NEUMANN
F. WOLF
K. YosHidA

\section{SUPPORTING INSTITUTIONS}

NIVERSITY OF BRITISH COLUMBIA

UNIVERSITY OF SOUTHERN CALIFORNIA

ALIFORNIA INSTITUTE OF TECHNOLOGY STANFORD UNIVERSITY

NIVERSITY OF CALIFORNIA

ONTANA STATE UNIVERSITY

UNIVERSITY OF HAWAII

NIVERSITY OF NEVADA

UNIVERSITY OF TOKYO

EW MEXICO STATE UNIVERSITY

UNIVERSITY OF UTAH

REGON STATE UNIVERSITY

NIVERSITY OF OREGON

WASHINGTON STATE UNIVERSITY

UNIVERSITY OF WASHINGTON

SAKA UNIVERSITY

AMERICAN MATHEMATICAL SOCIETY

The Supporting Institutions listed above contribute to the cost of publication of this Journal, but they a t owners or publishers and have no responsibility for its contents or policies.

Mathematical papers intended for publication in the Pacific Journal of Mathematics should be in typ Irm or offset-reproduced (not dittoed), double spaced with large margins. Underline Greek letters in re ierman in green, and script in blue. The first paragraph or two must be capable of being used separately as 'nopsis of the entire paper. Items of the bibliography should not be cited there unless absolutely necessary, hich case they must be identified by author and Journal, rather than by item number. Manuscripts, uplicate, may be sent to any one of the four editors. Please classify according to the scheme of Math. Revieu Idex to Vol. 39. All other communications should be addressed to the managing editor, or Elaine Bart Iniversity of California, Los Angeles, California, 90024.

100 reprints are provided free for each article, only if page charges have been substantially pai dditional copies may be obtained at cost in multiples of 50 .

The Pacific Journal of Mathematics is issued monthly as of January 1966. Regular subscription rate: \$72. year (6 Vols., 12 issues). Special rate: $\$ 36.00$ a year to individual members of supporting institutions.

Subscriptions, orders for numbers issued in the last three calendar years, and changes of address should ent to Pacific Journal of Mathematics, 103 Highland Boulevard, Berkeley, California, 94708.

UBLISHED BY PACIFIC JOURNAL OF MATHEMATICS, A NON-PROFIT CORPORATION

Printed at Jerusalem Academic Press, POB 2390, Jerusalem, Israel.

Copyright (C) 1978 Pacific Journal of Mathematics

All Rights Reserved 


\section{Pacific Journal of Mathematics}

\section{Vol. 75, No. $1 \quad$ September, 1978}

Mieczyslaw Altman, General solvability theorems

Denise Amar and Eric Amar, Sur les suites d'interpolation en plusieurs variables ..........................................

Herbert Stanley Bear, Jr. and Gerald Norman Hile, Algebras which satisfy a second order linear partial differential equation ..................

Marilyn Breen, Sets in $R^{d}$ having $(d-2)$-dimensional kernels ............

Gavin Brown and William Moran, Analytic discs in the maximal ideal space

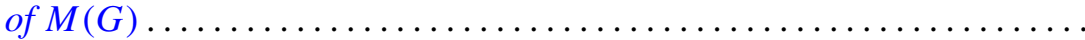

Ronald P. Brown, Quadratic forms with prescribed Stiefel-Whitney

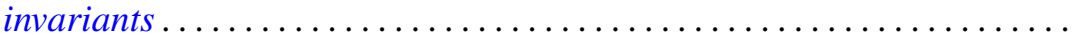

Gulbank D. Chakerian and H. Groemer, On coverings of Euclidean space by convex sets

S. Feigelstock and Z. Schlussel, Principal ideal and Noetherian groups.....

Ralph S. Freese and James Bryant Nation, Projective lattices ............

Harry Gingold, Uniqueness of linear boundary value problems for

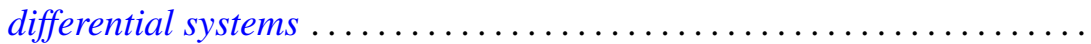

John R. Hedstrom and Evan Green Houston, Jr., Pseudo-valuation domains. . .

William Josephson, Coallocation between lattices with applications to measure extensions

M. Koskela, A characterization of non-negative matrix operators on $l^{p}$ to $l^{q}$ with $\infty>p \geq q>1$

Kurt Kreith and Charles Andrew Swanson, Conjugate points for nonlinear differential equations...........................

Shoji Kyuno, On prime gamma rings ........................ 185

Alois Andreas Lechicki, On bounded and subcontinuous multifunctions ..

Roberto Longo, A simple proof of the existence of modular automorphisms in approximately finite-dimensional von Neumann algebras ...

Kenneth Millett, Obstructions to pseudoisotopy implying isotopy for

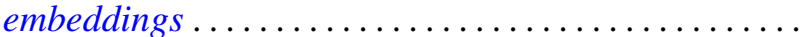

William F. Moss and John Piepenbrink, Positive solutions of elliptic equations. .

Mitsuru Nakai and Leo Sario, Duffin's function and Hadamard's

conjecture

Mohan S. Putcha, Word equations in some geometric semigroups ...

Walter Rudin, Peak-interpolation sets of class $C^{1} \ldots \ldots \ldots$

Elias Saab, On the Radon-Nikodým property in a class of locally convex

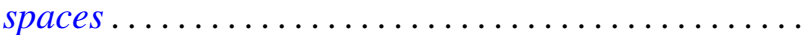

